\title{
Flocculation-ultrasonic assisted extraction and solid phase clean-up for determination of polycyclic aromatic hydrocarbons in water rich in colloidal particulate with high performance liquid chromatography and ultraviolet-fluorescence detection
}

\author{
Shun-Li Fan ${ }^{\text {a,b }}$, Lixia Zhao a, Jin-Ming Lin ${ }^{\text {a,* }}$ \\ ${ }^{a}$ State Key Laboratory of Environmental Chemistry and Ecotoxicology, Research Center for Eco-Environmental Sciences, \\ Chinese Academy of Sciences, P.O. Box 2871, Beijing 100085, China \\ ${ }^{\mathrm{b}}$ Henan Key Laboratory for Environmental Pollution Control, College of Chemistry and Environmental Sciences, \\ Henan Normal University, Xinxiang 453007, China
}

Received 23 November 2006; received in revised form 1 January 2007; accepted 14 January 2007

Available online 20 January 2007

\begin{abstract}
In the present work, a simple method of sample preparation for the determination of polycyclic aromatic hydrocarbons (PAHs) in water rich in colloidal particulate was developed. The technique was mainly based on the effect of the flocculation of aluminum sulfate and the adsorption of the flocculation aid florisil. The method contained three steps: flocculation, ultrasonic extraction, and solid-phase extraction cleanup. Major parameters of the procedure were optimized with high performance liquid chromatography (HPLC) separation and ultraviolet-fluorescence detection. When $250 \mathrm{~mL}$ model sample containing 16 EPA PAHs was processed, the developed method provided detection limits in the range of 0.02-5 ng/L. Both spiked and non-spiked polluted river water samples rich in suspended particles and organic matters were analyzed. Recoveries and relative standard deviations for the 16 PAHs were in ranges of $86-94 \%$ and $3-13 \%(n=5)$, respectively.
\end{abstract}

(c) 2007 Elsevier B.V. All rights reserved.

Keywords: Flocculation; Ultrasonic extraction; Solid-phase extraction; Polycyclic aromatic hydrocarbons; High performance liquid chromatography

\section{Introduction}

Polycyclic aromatic hydrocarbons (PAHs) are a special group of organic compounds originating from anthropogenic combustion processes and biogenic activities. Because of their mutagenic, carcinogenic, toxic nature and environmental ubiquitous occurrence, 16 PAHs have been selected as priority pollutants by the US Environmental Protection Agency (EPA) and consequently are monitored worldwide in environmental matrices. Although analytical instrumentations such as gas chromatography with mass spectrometry detection (GC/MS) and high performance liquid chromatography with fluorescence or ultraviolet detection (HPLC/FL or UV) have matured, determi-

\footnotetext{
* Corresponding author. Present address: Department of Chemistry, Tsinghua University, Beijing 100084, China. Tel.: +86 1062792343 ; fax: +86 1062792343

E-mail address: jmlin@ mail.tsinghua.edu.cn (J.-M. Lin).
}

nation of trace PAHs in aqueous samples is still often a difficult task as analytes enrichment and isolation are required. Sample preparation has become, in effect, a bottleneck in the laboratory processes nowadays [1,2].

Commonly used methods for aqueous PAHs sample preparation are liquid-liquid extraction (LLE) and solid-phase extraction (SPE) [1-6]. LLE has many well-known disadvantages such as emulsion and large volume consumption of toxic and flammable solvents, there is therefore an increasing tendency to replace LLE by SPE due to its advantages of selectivity of extraction and significant reduction of solvents consumption. SPE can be also used for solid samples by combination with other techniques like ultrasonic extraction [2,7,8]. However, SPE has drawbacks for water analysis, such as particle blockage, small cross-sectional area and slow sample processing rate, etc. [3]. It only works well with "clean" samples and has not yet been directly applied to polluted waters rich in colloidal particulate $[1,4,5]$. Furthermore, for water sample containing large amount 
of particles and colloidal matter, preliminary filtration of the sample before SPE is proved not only time consuming but also unreliability because considerable PAHs may be lost in the step owing to the rather intensive adsorption on the solid particulate matter [1].

In recent years, some new techniques including solid-phase micro-extraction (SPME) [9-11] and stir bar sorptive extraction (SBSE) [12-14] and liquid-phase micro-extraction (LPME) [15-17] have been developed rapidly. Since they are all equilibrium methods, based on the PAHs partitioning between the aqueous and the extraction phases, rather than exhaustive method like LLE or SPE, problems like extraction stability and sensitivity are often reported. In addition, there also remain other evident defects of them unsolved. For example, SPME has inherent disadvantage of severe sample carry-over between runs, and LPME usually suffers from interference of particles contained in the aqueous water $[15,16]$. Generally, these techniques are more suitable for determination of free PAHs in relatively "clean" water [18].

It is well-known that coagulation-flocculation process is widespread used for removal of colloidal particulate and organic matter in engineering of water or wastewater treatment. According to the literatures reported, with this process, $80 \%$ of organic compounds and at least $85 \%$ of suspended solid could be removed from water [19]. And additions of some adsorbents or flocculant aids such as activated carbon [20], organobenite [21], metal oxides [22] to the system may considerably promote the removal further due to the synergetic effect of the accelerated kinetics of coagulation-flocculation, sedimentation, and particularly of adsorption. To our knowledge, however, no work about using the coagulation-flocculation process as a sample preparation tool for the purpose of analytical specific organic compounds such as PAHs in water has been done.

Due to their hydrophobicity and poor solubility, PAHs present a great affinity for colloidal particles and organic matter in water and are partitioned a significant proportion in these matrices $[1,18]$. It can be expected that an appropriate coagulation-flocculation process will effectively "remove" PAHs from water into the floc sediment. In this study, an attempt at employing coagulation-flocculation process as one of the sample preparation steps for determination of EPA 16 PAHs from polluted water in rich colloidal particles has been made. In the process, PAHs in the water sample were first "removed" to the floc sediment using aluminum sulfate and florisil as coagulant and flocculant aid, respectively. Then, in combination with ultrasonic extraction and solid phase clean-up, PAHs were enriched from the sediment and were finally determined by HPLC with ultraviolet-fluorescence detection.

\section{Experiment}

\subsection{Reagents and materials}

The 16 PAHs, naphthalene (Nap), acenaphthylene (Acy), acenaphthene (Ace), fluorene (Flue), phenanthrene (Phe), anthracene (An), fluoranthene (Flut), pyrene (Py), benz $[a]$ anthra cene $(\mathrm{BaA})$, chrysene (Chy), benzo[b]fluoranthene $(\mathrm{BbF})$, benzo[ $k]$ fluoranthene $(\mathrm{BkF})$, benzo[ $[a]$ pyrene (Bap), dibenz $[a, h]$ anthracene (DBA), benzo[[a]]perylene (Bper), indeno[1,2,3-cd]pyrene (IP), were purchased from Aldrich (Milwaukee, WI, USA) and Supelco (Bellefonte, PA, USA). Individual stock solutions of these PAHs were made in acetonitrile at concentrations of approximately $250 \mu \mathrm{g} / \mathrm{mL}$. These solutions were used to make a PAHs mixture for chromatography experiment and sample spiking test. A standard solution of the 16 PAHs $(200 \mu \mathrm{g} / \mathrm{mL}$ for each compound) in methanol/methylene chloride $(1: 1, \mathrm{v} / \mathrm{v})$ was delivered from Accustandard (New Haven, CT, USA). HPLC grade acetonitrile and methanol were from Fisher (Fair Lawn, NJ, USA). Pesticide grade dichloromethane and acetone were obtained from Dikma (Beijing, China). Aluminum sulfate, sodium sulfate and ammonium hydroxide (25-28\%) were from Beijing Chemical Reagents Company (Beijing, China). Florisil (100-200 mesh) was from Jixiang Chemical Reagents Company (Shanghai, China). Sodium sulfate and Florisil were baked at 420 and $650{ }^{\circ} \mathrm{C}$, respectively, for at least $12 \mathrm{~h}$ and stored in seal containers. All other reagents were analytical grade and deionized ultrapure water (EasyPureTM LF, Barnstead, Iowa, USA) was used throughout. The glasswares were cleaned by successive acetone and dichloromethane rinsing, soaking in a detergent bath, water washing and stored at $120^{\circ} \mathrm{C}$ until use.

\subsection{Apparatus and method}

For the sample preparation, the procedures were mainly comprised three steps, flocculation, ultrasonication and SPE (Fig. 1).

In the flocculation step, a homemade conventional jar-test apparatus consisted of a beaker of $500 \mathrm{~mL}$ and an adjustable speed stirrer were employed. Coagulant of $6.0 \mathrm{~mL} 10 \%$ aluminum sulfate solution and flocculation aid of $4.0 \mathrm{~g}$ florisil were added directly to the beaker containing $250 \mathrm{~mL}$ water sample during the phase of agitation and the acidity was controlled at $\mathrm{pH} 6.0$ using ammonium hydroxide or hydrochloric acid. The solution was stirred rapidly at $150 \mathrm{rpm}$ for ca. $10 \mathrm{~s}$ during the reagents addition, followed by slow stirring at $60 \mathrm{rpm}$ for $20 \mathrm{~min}$ and quiescent settling for $15 \mathrm{~min}$. The supernatant was withdrawn as completely as possible by siphoning.

In the ultrasonication step, the precipitate was centrifuged at $3500 \mathrm{rpm}$ for $5 \mathrm{~min}$ with a TDL-40B centrifuge (Shanghai Anting Scientific Instrument Factory, Shanghai, China), then mixed with $8 \mathrm{~g}$ anhydrous sodium sulfate, extracted with $30 \mathrm{~mL}$ acetone-dichloromethane $(1: 1, \mathrm{v} / \mathrm{v})$ for $15 \mathrm{~min}$ at room temperature $\left(c a .25^{\circ} \mathrm{C}\right)$ in a ultrasonic bath $(40 \mathrm{kHz}$, Kunshan Ultrasonic Instrument Co., Jiangsu, China) with an occasional swirl to prevent its sticking to the bottom of the flask. The extract was filtered through a Whatman glass fiber filter and the filtrate was evaporated to half volume in a water bath so as to exclude the water-nonmiscible component of dichloromethane in the solvents.

The SPE was performed with Supelclean Envi-18 (3 mL) cartridges (Supelco, USA) on a set of Visiprep ${ }^{\mathrm{TM}}$ Solid Phase 


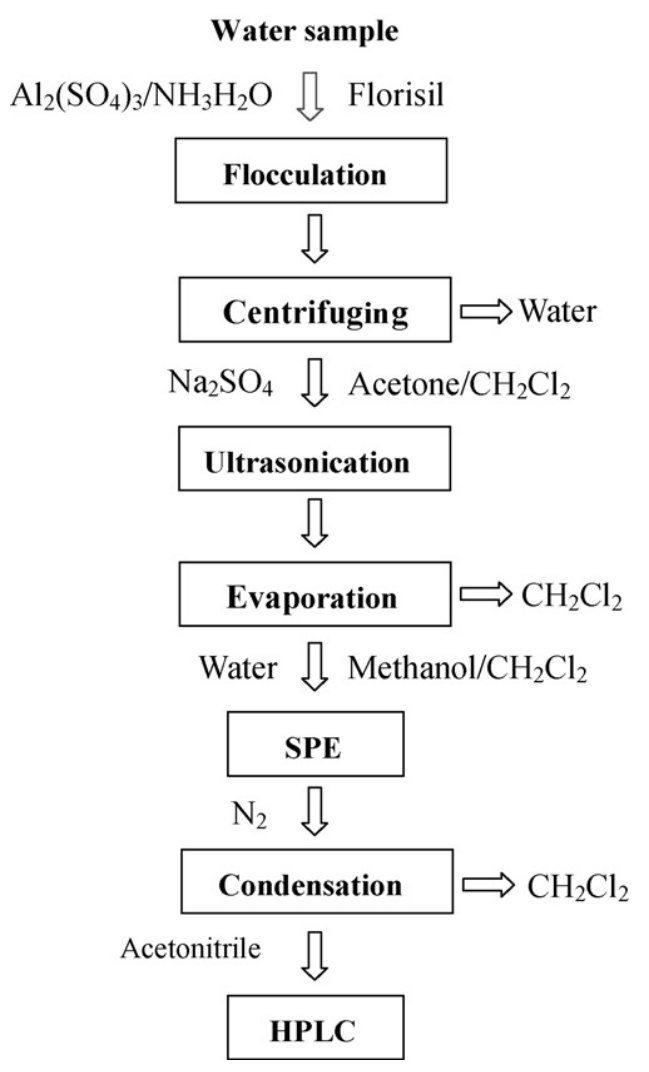

Fig. 1. Extraction procedures for the recovery of PAHs from waters.

Extraction Vacuum Manifolds (Supelco, USA). Prior to the SPE, the above solution obtained (about $15 \mathrm{~mL}$ ) was reconstituted by addition $35 \mathrm{~mL}$ of water to make the extraction solution to be $30 \%(\mathrm{v} / \mathrm{v})$ acetone in water. The SPE cartridge was conditioned with $5 \mathrm{~mL}$ dichloromethane, $5 \mathrm{~mL}$ acetone and $5 \mathrm{~mL}$ water in succession. The reconstituted solution was loaded at a flow rate of $4-6 \mathrm{~mL} / \mathrm{min}$. The cartridge was washed with $20 \mathrm{~mL} 40 \%$ acetone, dried by applying a vacuum ( $c a .1 \mathrm{~min}$ ) and passing through $0.3 \mathrm{~mL}$ methanol, then slowly eluted by application of $2.5 \mathrm{~mL}$ of dichloromethane. The eluate was evaporated to about $100 \mu \mathrm{L}$ under a gentle stream of ultrapure nitrogen and the final volume of the residue was adjusted to $1 \mathrm{~mL}$ with acetonitrile before HPLC analysis.

For the PAHs analyses, a Shimadzu HPLC-10Avp system (Shimadzu, Tokyo, Japan) equipped with time-programmable ultraviolet-fluorescence detectors and an automatic sample injector was used. The PAHs were separated in a Nucleosil-1005C18 PAH $(250 \mathrm{~mm} \times 4.6 \mathrm{~mm}$ ID) column (Macherey-Nagel, German). The chromatographic separation was performed with a mobile phase linear gradient comprising acetonitrile and water from $55 \%(\mathrm{v} / \mathrm{v})$ acetonitrile to $100 \%$ acetonitrile within $20 \mathrm{~min}$ and then the final composition was maintained for further $10 \mathrm{~min}$. The injection volume was $10 \mu \mathrm{L}$. The mobile phase flow rate was $1.3 \mathrm{~mL} / \mathrm{min}$ and the column temperature was controlled at $30^{\circ} \mathrm{C}$. Fifteen PAHs were detected by the FL detector except that Acy by UV due to the lack of fluorescence. The detection wavelength programme used is given in Table 1.
Table 1

Wavelength programing in UV and FL detection

\begin{tabular}{lllll}
\hline Time $(\min )$ & $\lambda_{\text {UV }}(\mathrm{nm})$ & $\lambda_{\text {ex }}(\mathrm{nm})$ & $\lambda_{\text {em }}(\mathrm{nm})$ & PAHs \\
\hline 0 & 254 & 296 & 430 & \\
8.40 & 220 & 280 & 340 & Nap \\
9.30 & 229 & & & Acy, Ace \\
11.45 & 254 & & & Flue \\
12.40 & & 250 & 350 & Phen \\
13.55 & & & 400 & An \\
14.76 & 235 & 280 & 450 & Flut \\
15.75 & 240 & & 393 & Py \\
18.00 & 287 & & 405 & BaA \\
19.57 & 267 & 268 & & Chy \\
21.24 & 256 & 296 & 430 & BbF \\
22.83 & 296 & & & BkF Bap \\
25.58 & & & 395 & DBA \\
27.00 & & & 418 & Bper \\
28.00 & 249 & & 500 & IP \\
\hline
\end{tabular}

\section{Results and discussion}

\subsection{Optimization of SPE procedure}

In the SPE of PAHs, some operational parameters such as conditioning of the cartridge, flow rate of the sample loaded were directly determined according to the cartridge manufacturer's advice without further optimization. Since the techniques of elution PAHs from the cartridge and dryness of the eluate have matured, the conditions of these used in this work mainly referenced the previous investigations with some minor modification [2]. Other parameters were investigated by using $100 \mathrm{~mL}$ mixing standard solution containing $0.5-50 \mathrm{ng}$ respective PAHs as a model sample. Each experiment was repeated three times and the data shown in the paper were the mean values.

One parameter studied was the concentration of the organic modifier added into the sample because the organic modifier could not only increase the solubility of PAHs thereby preventing them from adsorption onto the walls and surfaces of the system, but also keep the octadecyl chains of the $\mathrm{C}_{18}$ SPE cartridge in activated form [2]. In our experiments, acetone was used as the organic modifier. The recoveries of PAHs of the modified solution containing different percentages of acetone are shown in Fig. 2. The procedure for this investigation was

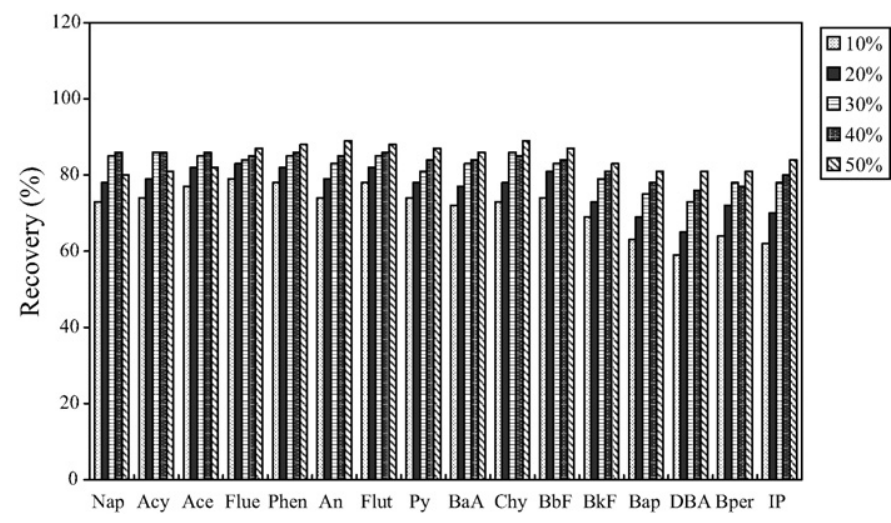

Fig. 2. Effect of acetone concentration on the recovery of PAHs. 
almost the same as that described in Section 2.2, except that the step of washing cartridge was omitted and the cartridge drying was carried out by only applying a vacuum $(20 \mathrm{~min})$. From Fig. 2 it is clear that the recoveries for PAHs increased with the increase of acetone concentration up to $40 \%$, which was especially more evident for the high molecular weight PAHs (5-6 rings). However, when the concentration of acetone was 50\%, the recoveries for the low molecular weight PAHs (2-3 rings) became lower. This can be simply explained by the increases of both solubility of PAHs and the eluotropic strength of the sample when the acetone content was increased. The increase of solubility of PAHs is mainly conducive to higher recoveries for the high molecular weight PAHs due to their more hydrophobicity, while the increase of the eluotropic strength resulted in smaller breakthrough volumes for the low molecular weight PAHs that have relatively high solubilities in water. Since the difference of the recoveries obtained with $30 \%$ and $40 \%$ acetone content was not significant, $30 \%$ acetone in the sample was used as an organic modifier in the following experiment.

A clean-up step is necessary before the analytes are eluted from the cartridge when real sample is considerably contaminated. According to the results of our experiments, $20 \mathrm{~mL} 40 \%$ acetone was suitable to be the solvent because it has eluotropic strength higher than that of the sample loaded and lower than that of the solvent (dichloromethane) used for eluting PAHs. More importantly, the results showed that this was the maximum concentration of acetone and amount used under the condition that the recoveries of the $16 \mathrm{PAHs}$ were in the acceptable ranges after the clean-up step. Therefore, $20 \mathrm{~mL} 40 \%$ acetone was used as SPE clean-up solvent in the real sample preparation.

After the cartridge clean-up, the cartridge drying is also an important aspect to be considered since an apolar solvent was used to elute the PAHs and the residue water on the cartridge can result in lower recovery and lower repeatability. Although water aspirator, flow of nitrogen or air, centrifuging are commonly used methods for drying the SPE cartridge nowadays, disadvantages are still present among them. All these methods are time consuming. Kiss et al. [6] found that about half amount of the residue water could be removed within $1 \mathrm{~min}$ with a water aspirator, but the other half seemed more refractory and still needed removal with nitrogen flow for $15 \mathrm{~min}$. Moreover, long time suction may result in the loss of volatile PAHs, trace impurity in nitrogen or air leads to interference in the following detection, centrifuging usually cannot remove the water utterly [6]. In this work, we tested a new method for dry the cartridge. The method is simply carried out by applying a vacuum for $1 \mathrm{~min}$ first, then passing through an appropriate amount of methanol to the cartridge. The influence of the amount of methanol on the PAHs recoveries was studied. The results (Fig. 3) showed that good recoveries (93-102\%) could be obtained when $0.3 \mathrm{~mL}$ methanol was applied. While Nap began to be eluted out and its recovery decreased significantly from $98 \%$ to $62 \%$ when $0.5 \mathrm{~mL}$ methanol was used. Compared with the results without applying methanol, it can be seen that methanol could efficiently remove the refractory water retained in the pores of the $\mathrm{C}_{18}$ sorbent due to its excellent miscibility.

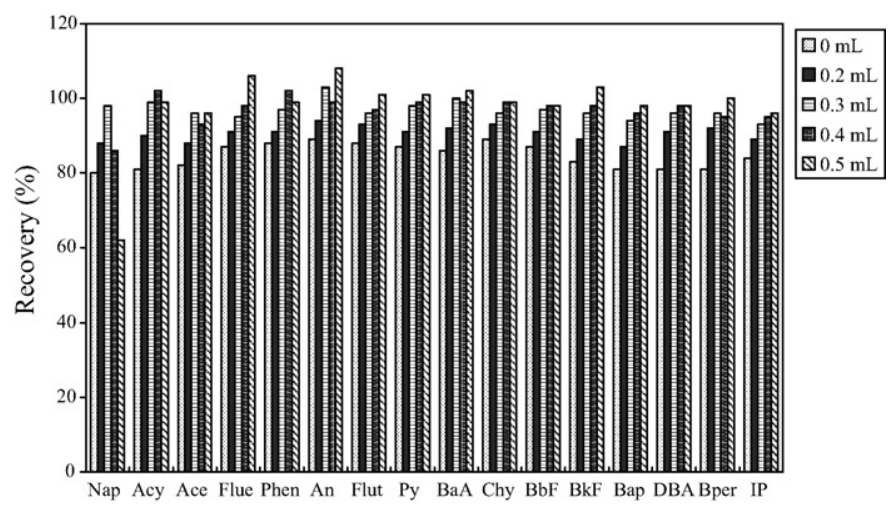

Fig. 3. Effect of cartridge drying with methanol on the recovery of PAHs.

\subsection{Optimization of ultrasonic extraction}

To optimize the ultrasonic extraction, the precipitate was initially obtained by flocculation of the model sample under almost the same conditions as that described in the first paragraph in Section 2.2, except that $2.0 \mathrm{~mL} \mathrm{Al} 2\left(\mathrm{SO}_{4}\right)_{3}(10 \%), 2.0 \mathrm{~g}$ florisil were used and slow stirring time was $10 \mathrm{~min}$. A mixture of acetone and dichloromethane $(1: 1, \mathrm{v} / \mathrm{v})$, which is often used as a solvent for ultrasonic extraction of PAHs from solid sample [23], was applied to the procedure. The effect of the amount of acetone-dichloromethane on the recoveries of PAHs was examined in the range of $20-40 \mathrm{~mL}$. As presented in Fig. $4,30 \mathrm{~mL}$ of acetone-dichloromethane could almost reach maximum recoveries $63-80 \%$ for all PAHs. More Consumption of solvent did not increase the recoveries significantly. So, $30 \mathrm{~mL}$ solvent of acetone-dichloromethane was chosen for the ultrasonic extraction.

\subsection{Optimization of flocculation procedure}

Flocculation is a two-step serial process that consists of destabilization of colloid particles or organic matter via charge neutralization by coagulant and agglomeration of destabilized particles into larger settleable flocs via sweep flocs, adsorption and inter-particle bridging. In this process it is essential that the coagulant agent be added by rapid mixing for dispersion it throughout the liquid and subsequently slow mixing encourages collision of the particles to agglomerate into larger flocs. More-

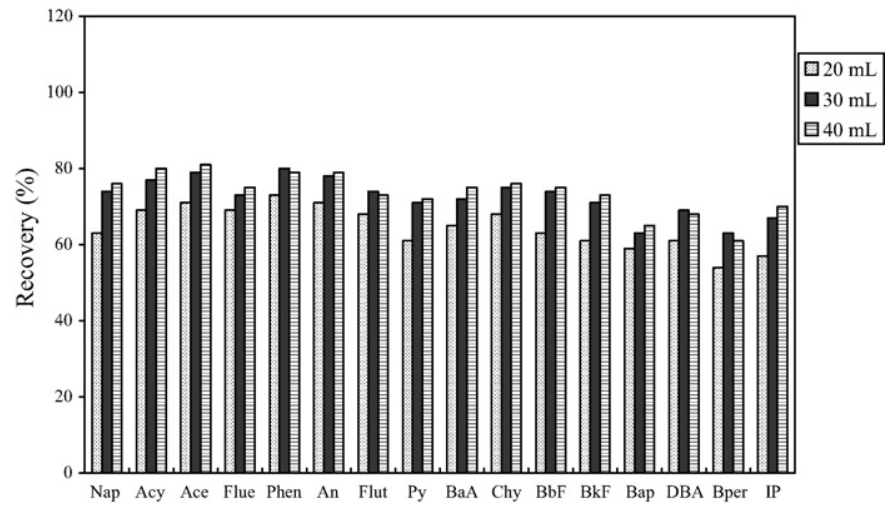

Fig. 4. Effect of solvent amount on the ultrasonic extraction of PAHs 


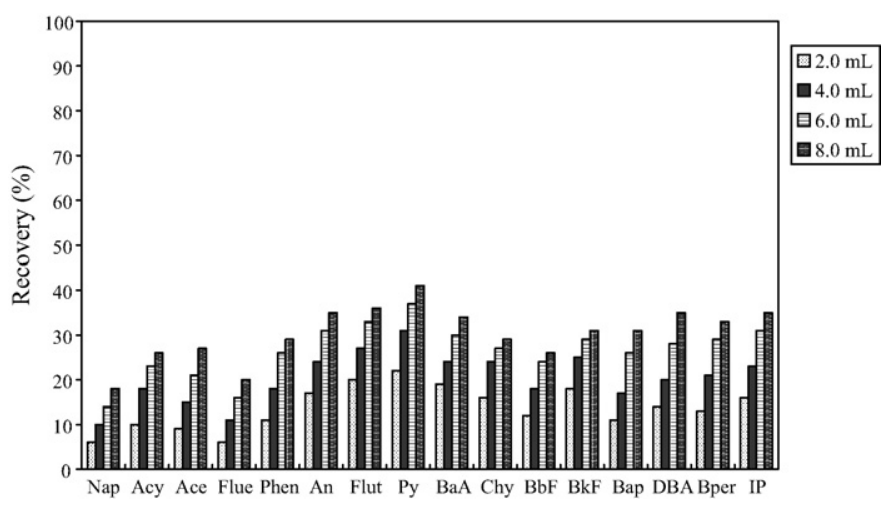

Fig. 5. Effect of $10 \%$ aluminum sulfate on the recovery of PAHs in the flocculation procedure.

over the dosages of the coagulants (including flocculation aid), $\mathrm{pH}$ of the liquid and flocculation time were important factors in removal of colloids and pollutants. The study was conducted with $250 \mathrm{~mL}$ water spiked with $16 \mathrm{PAH}(0.5-50 \mathrm{ng}$, respectively). And aluminum sulfate was selected as coagulant and florisil as flocculant aid due to their wide utilities in flocculation for wastewater treatment and adsorptive separation for analytical chemistry, respectively. The acidity of the liquid was always controlled at $\mathrm{pH} 6.0$ with ammonium hydroxide or hydrochloric acid according to the literature reported $\mathrm{pH} 5.5-8.0$ [24]. Optimizations of the dosage of coagulants and flocculation time were described bellow.

The result of the effect of the dosage of $10 \%$ aluminum sulfate is shown as Fig. 5. It can be seen that the efficiency of the PAHs recoveries was low when only aluminum sulfate was used as coagulant. Although the recoveries increased with the increase dosage added, product of hydrous aluminum oxide floc increased too correspondingly, and the floc settleablity was found to become poor when the coagulant dosage used was above $6.0 \mathrm{~mL}$. For the addition of $6.0 \mathrm{~mL}$ of $10 \%$ aluminum sulfate, the recoveries were $14-37 \%$, the precipitation time was $32 \mathrm{~min}$. To promote the efficiency of the flocculation, flocculation aid of florisil was added simultaneously with the coagulant of aluminum sulfate. The results (Fig. 6) revealed that addition of florisil not only significantly increased the recoveries but also saved the time of the precipitation. With the addition of $4.0 \mathrm{~g}$ florisil and $10 \mathrm{~min}$ slow stirring (60 rpm), almost maximum PAHs recoveries, 75-91\%,

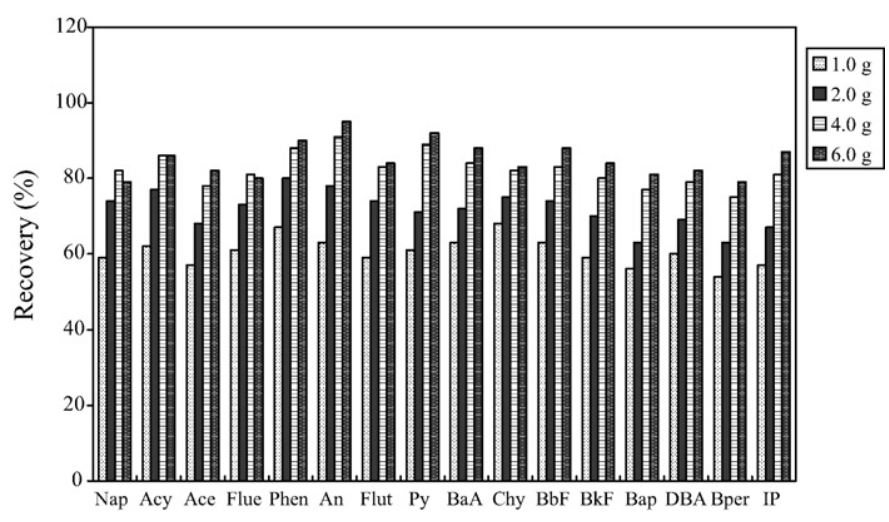

Fig. 6. Effect of florisil as flocculation aid on the recovery of PAHs.

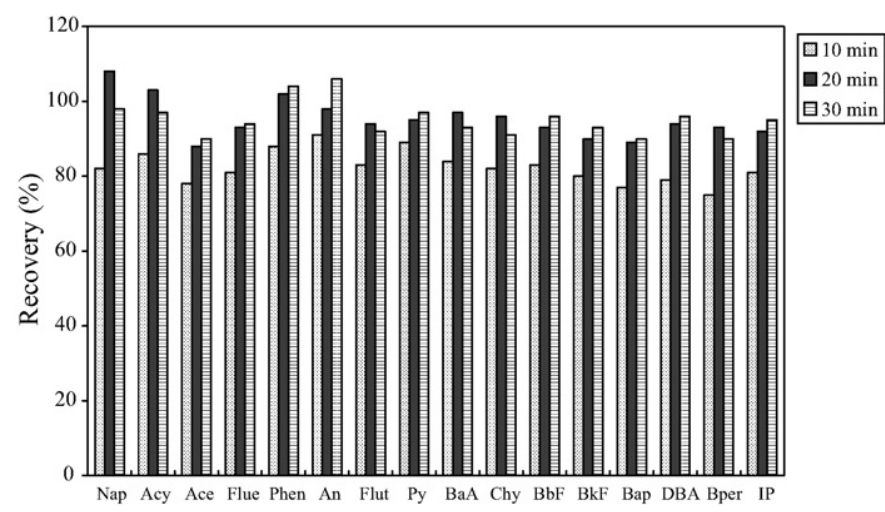

Fig. 7. Effect of floculation time on the recoveries of PAHs.

were yielded and the precipitation time was reduced to $15 \mathrm{~min}$. This performance suggested that florisil played an important part in removal of PAHs in the process.

The effect of floculation time on the recoveries of PAHs was examined in the range of 10-30 min with slow stirring operation. And the dosage of $10 \%$ aluminum sulfate and florisil were $6.0 \mathrm{~mL}$ and $4.0 \mathrm{~g}$, respectively. The results (Fig. 7) demonstrated that the recoveries increased with the increase of flocculation time in the range $10-20$ and $20 \mathrm{~min}$ flocculation provided almost the maximum recoveries (88-108\%), and 30 min flocculation did not give much difference with that. Comparing the results with that obtained under without use of florisil, we can conclude that most part of the PAHs was removed likely by florisil adsorption due to its large specific surface area and a small part by flocculation. Furthermore, the florisil particles might also serve as nuclei for the formation of flocs during the process, which improved the agglomeration of the flocs and consequently facilitated their settling if the fact that precipitation time was evidently reduced with the addition of florisil was considered.

In summary, the optimal condition of the flocculation procedure was that $6.0 \mathrm{~mL} 10 \%$ aluminum sulfate and $4.0 \mathrm{~g}$ florisil were added simultaneously into 250 water sample at $\mathrm{pH} 6.0$ with $c a .10 \mathrm{~s}$ of rapid mixing (150 rpm), $20 \mathrm{~min}$ of slow stirring $(60 \mathrm{rpm})$ for flocculation and then settled $15 \mathrm{~min}$ for precipitation.

\subsection{Analytical performance}

To understand the analytical performance of the proposed method, $250 \mathrm{~mL}$ of PAHs standard solutions covering each of their linear ranges were enriched and determined under the optimized conditions. Two typical chromatograms were shown as Fig. 8. Peak heights were used to evaluate the linearity, precision and sensitivity of the method and the results were presented in Table 2. Good linearity was observed over the ranges tested. The regression coefficients obtained were between 0.9908 and 0.9973. The relative standard deviations (R.S.D.) measured by five repeated determination of a standard solution containing $50 \mathrm{ng} / \mathrm{L}$ for each PAHs, except for An $25 \mathrm{ng} / \mathrm{L}$, ranged from $2 \%$ to $12 \%$. The limits of detection calculated with a signal to noise ratio of three were less than $0.7 \mathrm{ng} / \mathrm{L}$ for 14 PAHs and $5 \mathrm{ng} / \mathrm{L}$ and $3 \mathrm{ng} / \mathrm{L}$ for Acy and IP respectively. 

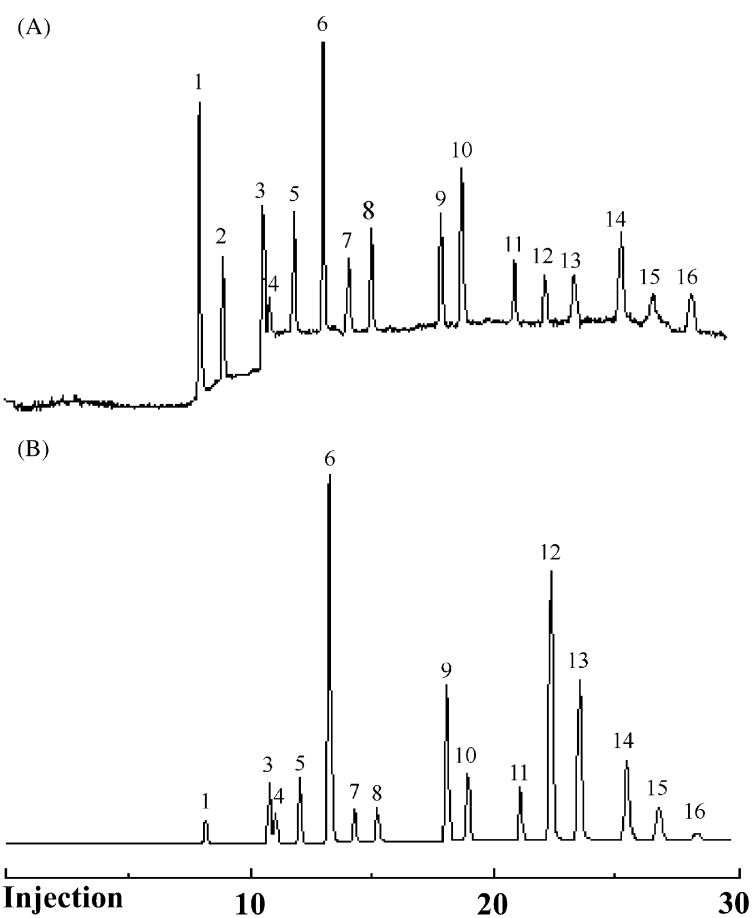

Fig. 8. Chromatograms for determination of 16 PAHs: (A) UV detection; (B) FL detection, $250 \mathrm{~mL}$ standard solution containing $50 \mathrm{ng} / \mathrm{L}$ for each PAHs enriched with the proposed method. 1: Nap; 2: Acy; 3: Ace; 4: Fue; 5: Phen; 6: An; 7: Flut; 8: Py; 9: BaA; 10: Chy; 11: BbF; 12: BkF; 13: Bap; 14: DBA; 15: Bper; 16: IP.

\subsection{Application to polluted water analysis}

The developed method had been applied to determine the 16 EPA PAHs in real water samples collected from the Qinghe River (Beijing, China). The water is polluted mainly with suspended particles and organic matters due to domestic wastewater dis-

Table 2

Some analytical characteristics of the proposed method

\begin{tabular}{lllrl}
\hline PAHs & $\begin{array}{l}\text { LODs }^{\text {a }} \\
(\mathrm{ng} / \mathrm{L})\end{array}$ & $\begin{array}{l}\text { R.S.D. } \\
(\%)\end{array}$ & $\begin{array}{l}\text { Range } \\
(\mathrm{ng} / \mathrm{L})\end{array}$ & $\begin{array}{l}\text { Regression } \\
\text { coefficient }\end{array}$ \\
\hline Nap & 0.7 & 12 & $5-500$ & 0.9916 \\
Acy & 5 & 9 & $20-500$ & 0.9923 \\
Ace & 0.5 & 5 & $2-100$ & 0.9958 \\
Flue & 0.5 & 6 & $2-100$ & 0.9968 \\
Phen & 0.2 & 3 & $1-100$ & 0.9945 \\
An & 0.02 & 7 & $0.1-50$ & 0.9921 \\
Flut & 0.2 & 4 & $1-100$ & 0.9937 \\
Py & 0.4 & 2 & $2-100$ & 0.9962 \\
BaA & 0.2 & 3 & $1-100$ & 0.9942 \\
Chy & 0.2 & 2 & $1-100$ & 0.9973 \\
BbF & 0.4 & 4 & $2-100$ & 0.9966 \\
BkF & 0.04 & 3 & $0.2-100$ & 0.9925 \\
Bap & 0.2 & 5 & $1-100$ & 0.9944 \\
DBA & 0.2 & 6 & $1-100$ & 0.9929 \\
Bper & 0.5 & 8 & $2-100$ & 0.9940 \\
IP & 3 & 9 & $10-500$ & 0.9908 \\
\hline
\end{tabular}

${ }^{\mathrm{a}}$ LOD: limit of detection(N/S = 3)

${ }^{b}$ R.S.D.: relative standard deviation, $50 \mathrm{ng} / \mathrm{L}$ for each PAHs, except for An $25 \mathrm{ng} / \mathrm{L}(n=5)$

${ }^{c}$ UV detection.
Table 3

Determination of PAHs in polluted water $(250 \mathrm{~mL}, n=5)$

\begin{tabular}{lllcll}
\hline PAHs & $\begin{array}{l}\text { Original found } \\
(\mathrm{ng} / \mathrm{L})\end{array}$ & $\begin{array}{l}\text { Added } \\
(\mathrm{ng} / \mathrm{L})\end{array}$ & $\begin{array}{l}\text { Total found } \\
(\mathrm{ng} / \mathrm{L})\end{array}$ & $\begin{array}{l}\text { Recovery } \\
(\%)\end{array}$ & $\begin{array}{l}\text { R.S.D. } \\
(\%)\end{array}$ \\
\hline Nap & 113 & 100 & 202 & 89 & 13 \\
Acy & $<$ DOL & 100 & 88 & 88 & 11 \\
Ace & 93 & 100 & 187 & 94 & 7 \\
Flue & 34 & 50 & 80 & 92 & 6 \\
Phen & 75 & 50 & 120 & 90 & 4 \\
An & 6 & 10 & 15 & 90 & 8 \\
Flut & 36 & 50 & 79 & 86 & 5 \\
Py & 34 & 50 & 77 & 86 & 3 \\
BaA & 37 & 50 & 81 & 88 & 4 \\
Chy & 64 & 50 & 107 & 86 & 3 \\
BbF & 69 & 50 & 112 & 86 & 4 \\
BkF & 57 & 50 & 101 & 88 & 4 \\
Bap & 30 & 50 & 73 & 86 & 5 \\
DBA & 52 & 50 & 95 & 86 & 9 \\
Bper & 56 & 50 & 101 & 90 & 9 \\
IP & 57 & 50 & 100 & 86 & 10 \\
\hline
\end{tabular}

${ }^{a} \mathrm{UV}$ detection.

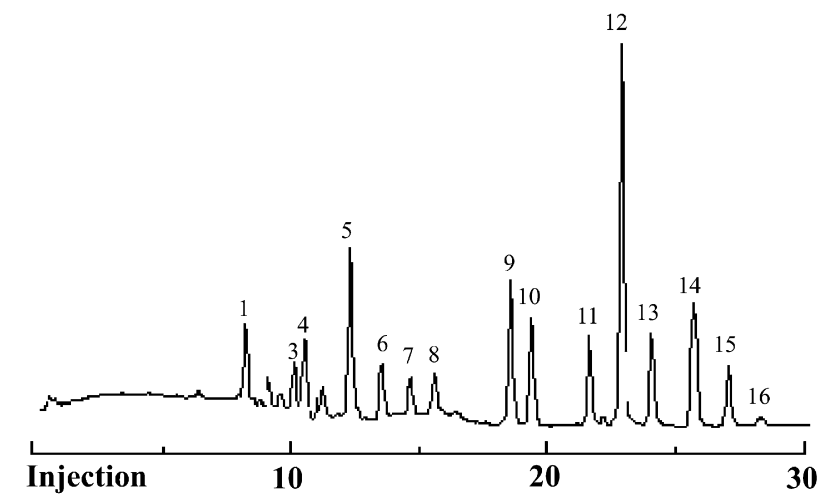

Fig. 9. HPLC-FL Chromatogram of polluted water sample concentrated by the proposed method, $250 \mathrm{~mL}$ river water was enriched under the optimal conditions. The peak numbers are same as in Fig. 8 .

charge and urban rainfall-runoff. Concentrations of suspended particles and organic matters in the water determined by standard gravimetric analysis and potassium dichromate oxidization method (CODcr) were 79.5 and $38.8 \mathrm{mg} / \mathrm{L}$, respectively. In order to validate the method, recoveries were also determined by spiking the sample with standard solution. The results are shown in Table 3. Chromatogram of the sample determined with FL detection is shown as Fig. 9. It can be seen that good recoveries (86-94\%) were obtained for 16 EPA PAHs. The relative standard deviations were in the range 3-13\% $(n=5)$. So, the developed method was applicable for determination of PAHs in water rich in colloidal particulate.

\section{Conclusions}

It was found that flocculation process could be used for recovery PAHs from polluted water rich in colloidal particulate. Employing aluminum sulfate as coagulant, florisil as flocculation aid, and combining with ultrasonic extraction, solid-phase clean-up techniques, we have studied the influence factors on the process and developed a new sample preparation method 
for determination of PAHs in water. The method was successfully applied to determine the 16 EPA PAHs in polluted river water using HPLC ultraviolet-fluorescence detection. Compared with SPE technique, the developed method has several advantages: Firstly, overcoming the problem of particle blockage often encountered by SPE. Secondly, saving time. In SPE, the time consumption is proportional to the volume of sample loaded, while flocculation is operated at a confined scale of time, thus, large sample volume (e.g. $>500 \mathrm{~mL}$ ) will make the process consume relatively less time provided that SPE should carry out the same task, although the whole time required by the current method (ca. $70 \mathrm{~min}$ ) is comparable with that of SPE. Finally, determination of total PAHs. The proposed method could be used for determination of total PAHs in very contaminated water, whereas SPE is usually used to determine dissolved or free PAHs in "clean" water and PAHs adsorbed on particles in water is excluded during the period of the sample preliminary filtration.

Although the experiments showed that the developed method was useful for water sample preparation, aluminum sulfate used only, as a coagulant, was proved not much effective for recovery of PAHs in water and a relative large amount of flocculation aid florisil was still required. Therefore, search and exploring new flocculation systems were needed to study further.

\section{Acknowledgements}

The authors gratefully acknowledge financial support of the National Natural Science Foundation of China (Nos. 20437020 and 20575073) and Major Research Program of Chinese Academy of Sciences (KZCX3-SW-432).

\section{References}

[1] E. Manoli, C. Samara, Trends Anal. Chem. 18 (1999) 417.

[2] R.M. Marce, F. Borrull, J. Chromatogr. A 885 (2000) 273.

[3] Z.S. Ferrera, C.P. Sanz, C.M. Santana, J.J.S. Rodriguez, Trends Anal. Chem. 23 (2004) 469.

[4] F. Busetti, A. Heitz, M. Cuomo, S. Badoer, P. Traverso, J. Chromatogr. A 1102 (2006) 104.

[5] E. Manoli, C. Samara, Chromatographia 43 (1996) 135.

[6] G. Kiss, Z. Varga-Puchony, J. Hlavay, J. Chromatogr. A 725 (1996) 261.

[7] Dugay, C. Herrenknecht, M. Czok, F. Guyon, N. Pages, J. Chromatogr. A 958 (2002) 1.

[8] F. Sun, D. Littlejohn, M.D. Gibson, Anal. Chim. Acta 364 (1998) 1.

[9] Mohammadi, Y. Yamini, N. Alizadeh, J. Chromatogr. A 1063 (2005) 1.

[10] P. Popp, C. Bauer, M. Moder, A. Paschke, J. Chromatogr. A 897 (2000) 153.

[11] R. Doong, S. Chang, Y. Sun, J. Chromatogr. A 879 (2000) 177.

[12] E. Baltussen, P. Sandra, F. David, C. Cramers, J. Microcol. Sep. 11 (1999) 737.

[13] B. Kolahgar, A. Hoffmann, A.C. Heiden, J. Chromatogr. A 963 (2002) 225.

[14] P. Popp, C. Bauer, L. Wennrich, Anal. Chim. Acta 436 (2001) 1.

[15] L. Zhao, H.K. Lee, Anal. Chem. 74 (2002) 2486.

[16] S. Shariati-Feizabadi, Y. Yamini, N. Bahramifar, Anal. Chim. Acta 489 (2003) 21.

[17] L. Hou, H.K. Lee, J. Chromatogr. A 976 (2002) 377.

[18] M.S. Garcia-Falcon, C. Perez-Lamela, J. Simal-Gandara, Anal. Chim. Acta 508 (2004) 177.

[19] M.I. Aguilar, J. Saez, M. Llorens, A. Soler, J.F. Ortuno, V. Meseguer, A. Fuentes, Chemosphere 58 (2005) 47.

[20] J.W. Lee, S.P. Choi, R. Thiruvenkatachari, W.G. Shim, H. Moon, Dyes Pigments 69 (2006) 196.

[21] Y.H. Shen, Environ. Technol. 23 (2002) 553

[22] L.O. Kolarik, Water Res. 17 (1983) 141.

[23] Y.F. Song, X. Jing, S. Fleischmann, B.-M. Wilke, Chemosphere 48 (2002) 993.

[24] T. Nandy, S. Shastry, P.P. Pathe, S.N. Kaul, Water, Air, Soil Pollut. 148 (2003) 15. 